ORIGINAL ARTICLE

\title{
Nasopharyngeal Carcinoma: A Retrospective Study on Imaging Patterns at Tikur Anbessa Specialized Hospital, Addis Ababa, Ethiopia
}

\author{
Amal Saleh Nour $^{1 *}$, Tequam Debebe Weldehawariat ${ }^{1}$, Aynalem Abraha \\ Woldemariam $^{1}$, Dheresa Gelana Layo ${ }^{2}$
}

\footnotetext{
OPEN ACCESS

Citation: Amal Saleh, Tequam Debebe,

Aynalem Abraha, Dheresa Gelana.

Nasopharyngeal Carcinoma: A

Retrospective Study on Imaging Patterns

at Tikur Anbessa Specialized Hospital,

Addis Ababa, Ethiopia. Ethiop J Health

Sci.2020;30(2):215.

doi:http://dx.doi.org/10.4314/ejhs.v30 i2.9

Received: October 01, 2019

Accepted: November 18, 2019

Published: March 1, 2020

Copyright: (C2020 Amal S., et al. This is an open access article distributed under the terms of the Creative Commons Attribution License, which permits unrestricted use, distribution, and reproduction in any medium, provided the original author and source are credited.

Funding: Nil

Competing Interests: The authors declare that this manuscript was approved by all authors in its form and that no competing interest exists.

Affiliation and Correspondence:

${ }^{1}$ Department of Radiology, College of

Health sciences, Addis Ababa

University, Addis Ababa, Ethiopia.

${ }^{2}$ Department of Radiology, Jimma

University College of Health Sciences,

Ethiopia.

*Email: salehamal12@gmail.com
}

\begin{abstract}
BACKGROUND: Nasopharyngeal carcinoma is one of the rare forms of cancer globally which has a high incidence in select geographic and ethnic populations. The current study aims to assess the clinical presentation, imaging patterns and radiologypathology correlation of nasopharyngeal carcinoma.

METHODS: This is a retrospective analysis of 80 patients with newly diagnosed nasopharyngeal carcinoma who came to Tikur Anbessa Specialized Hospital from January 2016 to August 2017. The patients' history, physical examination, abdominopelvic ultrasound, chest $x$-ray, computed tomography scan and biopsy reports were reviewed from their medical record. Patients who had previous history of treatment for nasopharyngeal carcinoma, those who did not have biopsy and those without imaging were excluded from the study.

RESULTS: This study showed that 61(81.3\%) patients had neck swelling as the initial clinical symptom, and the average time of presentation of symptoms was 6(IQR 8) months. On the other hand, 56(70.0\%) were diagnosed with non-keratinizing undifferentiated nasopharyngeal carcinoma while 15(3.8\%) had keratinizing nasopharyngeal carcinoma. Sixty-nine (86.3\%) patients had nodal metastasis; $22.5 \%$ had invasion into the paranasal sinuses; $47.5 \%$ had T4 with $\mathrm{T} 1, \mathrm{~T} 2$ and $\mathrm{T} 3 \mathrm{being} 18.8 \%$, $17.5 \%$ and $7.5 \%$ stage respectively at time of diagnosis. Ninety percent of the cases had a diagnosis of nasopharyngeal carcinoma on imaging, but $10 \%$ were given alternative diagnosis.

CONCLUSION: Non-keratinizing undifferentiated type was the commonest histologic subtype in this study which is also recognized as the commonest one in endemic countries. A significant number did not have a specific histologic WHO type on pathology report which could in turn affect the management and assessment of risk factors.

KEYWORDS: Nasopharyngeal carcinoma, CT imaging pattern, Histopathology
\end{abstract}




\section{INTRODUCTION}

Nasopharyngeal carcinoma (NPC) is a cancer that arises from the nasopharynx epithelium with the epicenter of the mass at the fossa of Rosenmüller (1). The clinical behavior, epidemiology, and histopathology are different from that of squamous cell carcinomas of the head and neck $(1,2)$.

On the basis of the World Health Organization (WHO) classification, NPC is classified into three pathological subtypes. Keratinizing squamous cell carcinoma (formerly WHO type I), nonkeratinizing carcinoma which is further subclassified as differentiated and undifferentiated (formerly WHO Type II and III). The final subtype is Basaloid squamous cell carcinoma (3).

There are increased risks associated with certain foods eaten within high-risk areas, such as salted fish, certain preserved foods, and hot spices, which are high in nitrosamine compounds and volatile nitrosamines (4). Patients present with local and non-specific symptoms, such as neck swelling and epistaxis, depending on the route of spread and anatomic structures affected including hearing loss, otalgia, headache, or cranial nerve palsy $(1,2)$.

Because of the location of the disease, nonspecific clinical presentation and the difficulty to do clinical examination of the nasopharynx, patients are often misdiagnosed and present with advanced stage $(5,6)$. Computed tomography $(\mathrm{CT})$ and Magnetic resonance imaging (MRI) are the recommended imaging modalities of choice $(1,7)$. The tumor, node and metastasis (TNM) classification of the American Joint Committee on Cancer (AJCC) $7^{\text {th }}$ is used to determine the tumor staging. A definitive diagnosis of NPC needs a positive biopsy, and the role of imaging is for staging purposes. As to our knowledge, there is no study done on NPC in Ethiopia, and the burden of the disease is not known. The intention of this study is to assess the clinical presentation, imaging patterns and pathology subtype of nasopharyngeal carcinoma.

\section{METHODS}

The study was conducted from January 2016 to August 2017 at Tikur Anbessa Specialized Hospital (TASH), Addis Ababa, Ethiopia. It is a tertiary hospital and the only cancer center that provides radiotherapy for its patients. The Department of Radiology in the hospital has advanced imaging modalities, such as a $1.5 \mathrm{~T}$ magnetic resonance imaging (MRI), a 128 and 64 slice computed tomography (CT) machines. This was a retrospective study conducted by reviewing the medical records of all newly diagnosed, untreated nasopharyngeal carcinoma patients who came to the oncology department at TASH. The medical records of 92 patients were reviewed, 4 were mislabeled as NPC and 8 had incomplete data leaving 80 patients. The patients' history, physical examination, abdominopelvic ultrasound, chest $\mathrm{x}$ ray, computed tomography scan and biopsy reports were reviewed by the principal investigator. Patients who had previous history of treatment for NPC, those who did not have biopsy and those without imaging were excluded from the study. The biopsy was taken from the primary nasopharyngeal lesion or from involved neck lymph nodes. The reviewed CT scans were from TASH as well as private institutions. All data were recorded on a structured questionnaire. The patients' imaging reports were analyzed and restaged according to the American Joint Committee on Cancer (AJCC) $7^{\text {th }}$ edition for nasopharyngeal carcinoma(3). Patients who did not have CT, biopsy or had incomplete medicals record were excluded from the study.

Ethical consideration: Ethical approval was obtained from the Ethics Committee of the Department of Radiology before the commencement of the study.

Statistical analysis: Mean and ranges were calculated for continuous variables. Proportions and frequency tables were used to summarize categorical variables. Statistical analysis was done using statistical package for social sciences 21 (SPSS). Fisher's exact tests was used to assess the significance of association between independent and dependent variables.

\section{RESULTS}

In this study, there were a total of 80 patients; $54(67.5 \%)$ were males while 26(32.5\%) were females, with male-to-female ratio of $2: 1$. The mean age was $37.3 \pm 16.2$ years ranging from 13 to 78 years. The age group of 21 to 30 years was $24(30 \%)$ as shown in Figure 1. 


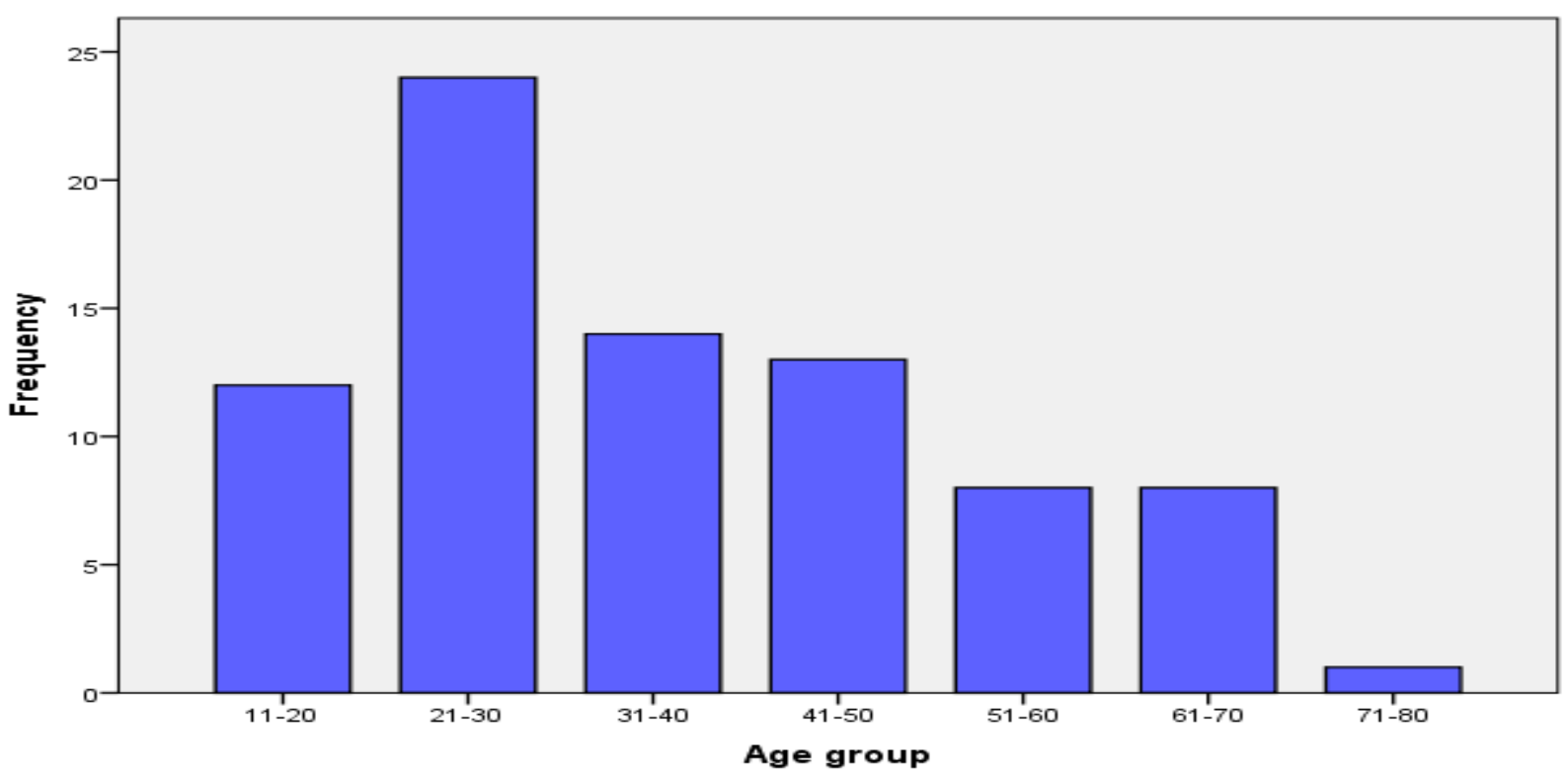

Figure 1: Age group distribution of patients diagnosed with NPC at Tikur Anbessa Hospital, January 2016-August 2017

Patients had multiple clinical presentations at the time of diagnosis. Neck swelling as the initial clinical symptoms was seen in $61(81.3 \%)$ patients with $19(23.8 \%)$ having bilateral and 46(57.5\%) having unilateral neck swelling. Nasal obstruction was seen in 32(40\%) patients and 19 had headache as listed in Table 2.

Table 1: Clinical Symptoms of patients diagnosed with NPC at Tikur Anbessa Hospital, January 2016- August 2017.

\begin{tabular}{lrr}
\hline Clinical Symptoms & Frequency (n) & Percent (\%) \\
\hline Bilateral Neck Swelling & 19 & 23.8 \\
Unilateral Neck Swelling & 46 & 57.5 \\
Headache & 19 & 23.8 \\
Dysphagia & 6 & 7.5 \\
Nasal Obstruction & 32 & 40 \\
Hearing Complaint & 15 & 18.8 \\
Cranial Nerve Involvement & 4 & 5 \\
Voice Change & 3 & 3.8 \\
Others & 19 & 23.8 homogenous \\
average time of symptom presentation was six & enhancement. In 25.0 \%, the enhancement pattern \\
ths (IQR 8) ranging from six months to 24 & was not specified. Skull base invasion was seen in \\
ths with 23.8\% presenting at 12 months. & 47 (58.8\%) and intracranial and cavernous \\
rogeneous CT contrast enhancement pattern & extension was seen in 28 (35.0\%) and 15 (18.8\%) \\
seen in 40.0\% of lesions while 31.3\% had & respectively. Invasion into the paranasal sinuses 18
\end{tabular}


cases (22.5\%), the sphenoid sinus alone was involved in seven cases. Sphenoid sinus and/or additional ethmoid, maxillary and frontal sinus involvement was seen in five $(6.3 \%)$ patients.
Perineural spread to foramen ovale was seen in 12 cases $(15 \%)$. Perivascular spread through the carotid canal was seen in three patients $(3.8 \%)$ as seen in Table 2.

Table 2: Imaging findings of patients diagnosed with NPC at Tikur Anbessa Hospital January 2016-

August 2017

Characteristics

CT Contrast Patterns

Homogenous

Heterogeneous

Enhancing

Not specified

Intracranial

Cavernous Sinus

Middle Cranial

Posterior Cranial

Anterior Cranial

Perineural Involvement

Foramen Ovale

Foramen Spinosum

Foramen Lacerum

Hypoglossal Canal

Vidian Canal
Frequency

$\begin{array}{ll}25 & 31.3 \\ 32 & 40 \\ 3 & 3.8 \\ 20 & 25 \\ & \\ 15 & 18.8 \\ 11 & 13.8 \\ 6 & 7.5 \\ 4 & 5.0 \\ & \\ 12 & 15.0 \\ 6 & 7.5 \\ 9 & 11.3 \\ 2 & 2.5 \\ 3 & 3.8\end{array}$

According to the $\mathrm{T}$ stage, 38 patients $(47.5 \%)$ had T4 with T1, T2 and T3 being $18.8 \%, 17.5 \%$ and $7.5 \%$ respectively. In $7(8.8 \%)$ patients, staging could not be done. Nodal metastasis was seen in 69 out of $80(86.3 \%)$ patients with $42.5 \%$ having N2 stage and $16.3 \%$ having N1 stage. In $31(38.8 \%)$ of patients, the nodal stage could not be assessed. Central necrosis of lymph nodes were seen in 26 patients. The overall staging is summarized in Table
Percent

31.3

3.8

18.8

7.5

5.0

11.3

3.8

Table 3: Staging of patients diagnosed with NPC at Tikur Anbessa Hospital January 2016- August 2017.

\begin{tabular}{lll}
\hline Variable & Frequency & Percent \\
\hline Staging & 3 & \\
Stage 1 & 6 & 3.8 \\
Stage 2 & 13 & 7.5 \\
Stage 3 & 20 & 16.3 \\
Stage 4A & 3 & 25.0 \\
Stage 4B & 8 & 3.8 \\
Stage 4C & 27 & 10.0 \\
Not specified & & 33.8 \\
Histologic Finding & 3 & \\
Keratinizing SCC & 6 & 3.8 \\
Non-keratinizing differentiated & 56 & 7.5 \\
Non-keratinizing undifferentiated & 15 & 70.0 \\
NPC without histologic classification & 15.8 \\
\hline
\end{tabular}

DOI: http://dx.doi.org/10.4314/ejhs.v30i2.9 
Figure 2 is a CT image showing an example of the pattern of spread, involvement of the skull base and lymphadenopathy in one of the patients included in this study. Histologic diagnosis of solely nasopharyngeal carcinoma, without specific subtype was seen in $15(18.8 \%)$ patients while 65 patients were given specific histologic diagnosis out of which $56(70.0 \%)$ were non-keratinizing undifferentiated NPC. There was distant metastasis to the liver in seven $(8.8 \%), 2(2.5 \%)$ to the lungs and three $(3.8 \%)$ to the bones. There was no significant association seen between clinical presentation duration and the TNM staging $(\mathrm{P}=$ $0.38)$.
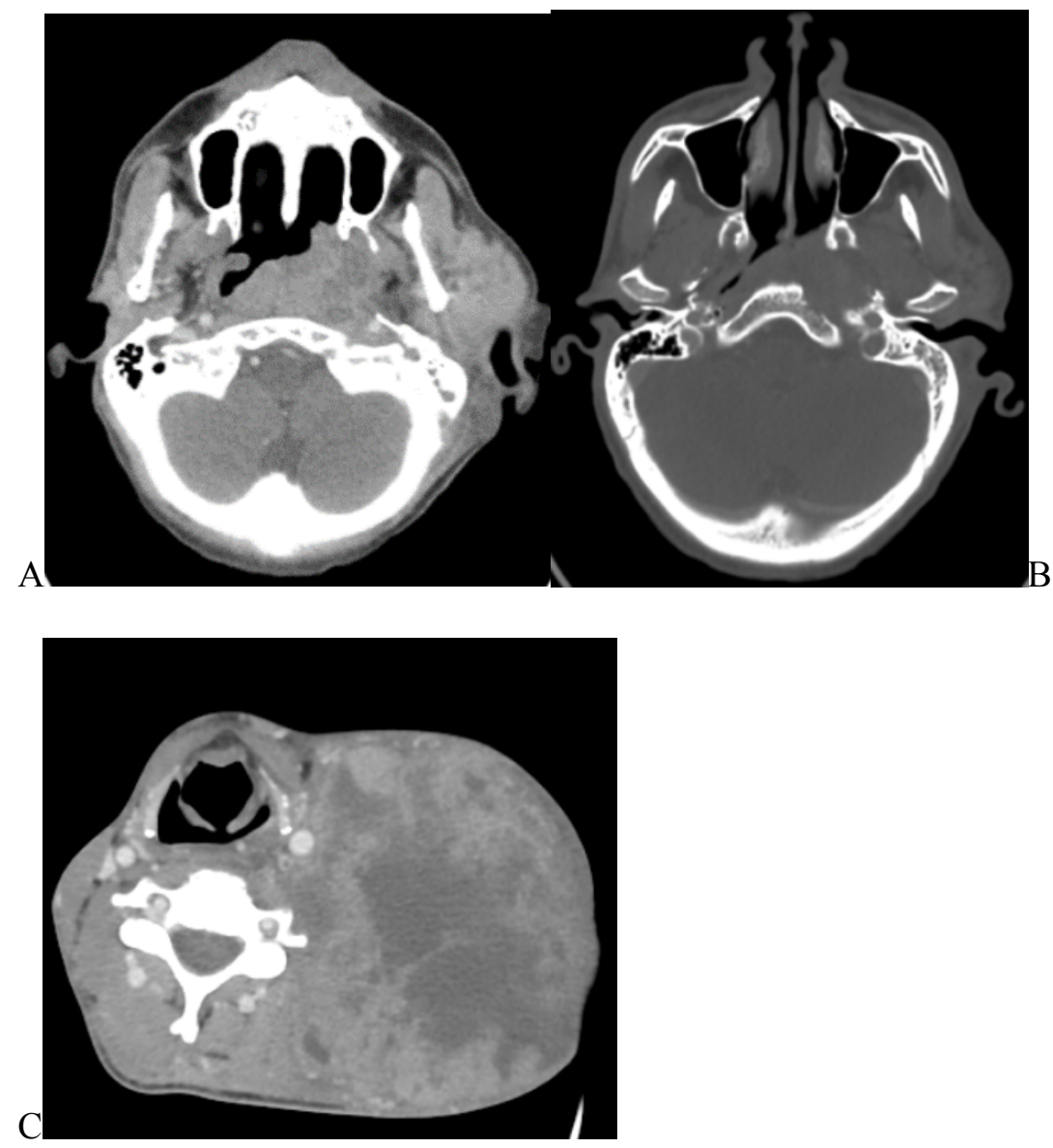
a) post-contrast axial CT
b) Axial bone window
c) Post contrast axial CT

Figure 2 Shows Axial post contrast soft tissue and bone window CT images of a 20-year-old male patient with undifferentiated NPC who presented with left neck swelling, ear pain and dysphagia of 7 months' duration. Left nasopharyngeal mass with extension to the parapharyngeal and masticator space (a) with erosion of the petrous bone and left side of the clivus (b) and large necrotic left level III lymph node (c). 


\section{DISCUSSION}

As to my knowledge, there is no study done on NPC in Ethiopia. The intention of this study is to assess the clinical presentation, imaging patterns and pathologic subtype of NPC. In this study, NPC was twice as more common in males than in females, which is consistent with data stated in the several papers and the global cancer statistics $(1,4,8)$. The bimodal age presentation of NPC is not seen in this study; rather, a peak at 31 to 30 age group was identified.

Most patients had multiple clinical presentations at the time of diagnosis with the commonest being neck swelling which is in line with several researches done by Parsad et-al and Abdullah et-al $(9,10)$. Nasal obstruction and headache were common, which is consistent with other researches where the clinical presentation is often a combination of the presence of mass, dysfunction caused by eustachian tube, skull base erosion and upper neck mass as described by Wei, W.I. $(1,5)$.

A delay in presentation seen in our patients has also been mentioned by Prasad et-al with several contributing factors ranging from lack of awareness by both patients and consulted physicians as well as the non-specific symptoms contributing to late presentation to health facilities as stated by Wei et-al $(5,6,9)$. This is even more complicated in Ethiopia where Tuberculosis (TB) is endemic and cervical lymph node is the commonest site to be involved in TB lymphadenitis causing further delay in the correct diagnosis $(11,12)$.

According to the $\mathrm{T}$ stage, the majority had T4 in our study, and T3 was significantly lower. Tstaging could not be done for a significant number of cases because of lack of adequate information on CT reports. This in turn plays a substantial role in the $T$ staging as well as overall staging which could explain why the $\mathrm{T} 3$ stage in this study was much lower compared to findings reported elsewhere. This inaccurate staging in turn will affect the patient management and assessment of interval change on imaging once treatment is started. The treatment plan changes once a tumor is designated as T2 (or more) and not T1, as adjuvant chemotherapy is added to the treatment regimen (13).

A significant number of patients had nodal metastasis. In approximately one third of patients, the nodal stage could not be assessed which also compromised the staging because of inadequate information on imaging reports. The evaluation of the status of lymph nodes is extremely important and physical examination alone is said to be inaccurate (12). The histologic status of a lymph node on CT by using criteria such as central necrosis or node size larger than $1 \mathrm{~cm}$ has been shown to be dependable (14).

Skull base invasion was common and was seen in over half the cases and overall intracranial extension was higher compared to research done by Sham et-al. The intracranial extension to the middle cranial fossa was comparable with Sham etal (15). Paranasal sinus invasion in our study was in line with research done by Li Tian et al (16) with the frequently involved sinus being the sphenoid sinus.

Perineural spread especially through foramen ovale was the commonest finding. The commonest WHO subtype was non-keratinizing undifferentiated NPC. There were only a few cases of keratinizing differentiated type in this study. As stated by Glastonbury et-al, non-keratinizing NPC is said to be the most common form, approximately $75 \%$, with undifferentiated type being around 4 times more common than differentiated type (13).

This was also reported by BS Alabi et al where $70 \%$ were non-keratinizing undifferentiated subtype in Ilorin, Nigeria (17). This histologic type of NPC is commonly associated with tumors found in endemic countries. These findings are consistent with our study. Pathology reports stated simply as NPC as the final diagnosis without specific subtype is not the reporting format recommended by WHO. This could potentially mean that a larger number of patients could have non-keratinizing undifferentiated nasopharyngeal carcinoma raising the question of whether it is endemic in Ethiopia. This is a daunting piece of information as a great deal is being done where NPC is endemic in terms of possible screening, treatment modality and follow-up for a better outcome in these patients.

Although biopsy is the gold standard to make a diagnosis, imaging can recommend a different

DOI: http://dx.doi.org/10.4314/ejhs.v30i2.9 
differential diagnosis particularly based on the site of origin of the lesion. Nasopharyngeal carcinoma is a cancer that arises from the nasopharynx epithelium with the epicenter of the mass at the fossa of Rosenmüller. Possible differential diagnosis of malignant tumors of the nasopharynx includes lymphoma, extramedullary plasmacytoma, adenoid cystic carcinoma, melanoma and rhabdomyosarcoma (1).

The limitation of this study was that this was a retrospective study; therefore, there was no control over the amount of information that could be gathered. Epstein Barr virus (EBV) has been studied as an etiologic factor, especially in the endemic type, and is considered an important step in NPC progression (18). There was no Epstein Barr virus (EBV) detection done in this study, which would have further strengthened the ability to assess if there is any correlation between EBV and NPC in Ethiopia. The biopsy was taken from lymph nodes or the nasopharyngeal mass rather than the recommended biopsy from the nasopharyngeal mass itself. The pathology reports did not follow WHO regulations.

In conclusion, nasopharyngeal carcinoma is an important form of cancer that emphasis should be put on in Ethiopia. The commonest presentation was cervical lymphadenopathy with advanced stage. Non-keratinizing undifferentiated type was the commonest histology raising the possibility that NPC could be endemic in Ethiopia. A substantial number did not have a specific histologic WHO sub-type on pathology report.

It is the recommendation of this study that radiologists need to report their imaging findings with staging done according to AJCC, and pathologists should use the standard WHO classification in their reports. TB is endemic in Ethiopia, but it is important to differentiate TB lymphadenitis from metastatic lymphadenopathy in order to prevent delay in diagnosis of NPC. This study shows that the histologic type is one that is found in endemic regions. We recommend that a prospective study be conducted to determine the possible causes and risk factors for NPC in the Ethiopian population.

\section{REFERENCES}

1. Chua M.L.K., Wee J.T.S, Hui E.P, Chan A.T.C. Nasopharyngeal carcinoma: Lancet: 2016; 387(10022): 1012-1024.

2. Ahmed Abdel Khalek Abdel Razek, A. King. MRI and CT of Nasopharyngeal Carcinoma. AJR, 2012; 198(1):11-8.

3. Edge S.B., C.C. Compton. The American Joint Committee on Cancer: the 7th edition of the AJCC cancer staging manual and the future of TNM: Ann Surg Oncol, 2010; 17(6): 1471-4.

4. Torre L.A., Bray F, Siegel R.L, Ferlay J., Lortet-Tieulent J., Jemal A. Global cancer statistics: 2012. CA: A Cancer Journal for Clinicians: 2015: 65(2): 87-108.

5. Wei W.I, J.S.T. Sham. Nasopharyngeal carcinoma: The Lancet, 2005; 365(9476): 2041-2054.

6. Kataki A.C. et al.: Nasopharyngeal carcinoma in the Northeastern states of India: Chin $J$ Cancer: 2011: 30(2): 106-13.

7. Stambuk H.E., Patel S.G, Mosier K.M, Wolden S.L, Holodny A.I. Nasopharyngeal carcinoma, recognizing the radiographic features in children: AJNR Am J Neuroradiol: 2005: 26(6): 1575-9.

8. Jia W.H., Huang Q.H., Liao J., et al.: Trends in incidence and mortality of nasopharyngeal carcinoma over a 20-25 year period (1978/1983-2002) in Sihui and Cangwu counties in southern China: BMC Cancer: 2006: 6 (1): 178

9. Prasad U., K.C. Pua: Nasopharyngeal carcinoma, a delay in diagnosis: Med $J$ Malaysia: 2000: 55(2): 230-5.

10. Abdullah N.E., Adam A.A.M., Khalifa E.H., et al: Nasopharyngeal Cancer in Sudan: Epidemiology, Clinical and Histological Characteristics: Clinical Medicine Insights, Ear, Nose and Throat: 2011: 4 (CMENT): S5825.

11. Bezabih M., D.W. Mariam, S.G. Selassie. Fine needle aspiration cytology of suspected tuberculous lymphadenitis. Cytopathology, 2002; 13(5): 284-90.

12. Muluye D., Biadgo B., Woldegirma E., Ambachew A. Prevalence of tuberculous 
lymphadenitis in Gondar University Hospital, Northwest Ethiopia: BMC Public Health: 2013: 13 (1): 435-435.

13. Glastonbury, C.M. K.L. Salzman. Pitfalls in the staging of cancer of nasopharyngeal carcinoma. Neuroimaging Clin $N \mathrm{Am}$, 2013;23(1): 9-25.

14. Friedman M. et al: Nodal size of metastatic squamous cell carcinoma of the neck: Laryngoscope: 1993: 103(8): 854-6.

15. Sham J.S., Cheung Y.K, Choy D., Chan F.L, Leong L. Nasopharyngeal carcinoma: CT evaluation of patterns of tumor spread: $A J N R$ Am J Neuroradiol: 1991. 12(2): 265-70.
16. Tian L., Li Y.Z, Mo Y.X., et al: Nasopharyngeal carcinoma with paranasal sinus invasion, the prognostic significance and the evidence-based study basis of its T-staging category according to the AJCC staging syste: BMC Cancer: 2014: 14 (1): 832

17. BS Alabi, KB Badmos, OA Afolabi, MO Buhari: Clinico-Pathological pattern of nasopharyngeal carcinoma in Ilorin, Nigeria: Nigerian Journal of Clinical Practice: 2010 13 (4): 445-448.

18. Brennan, B., Nasopharyngeal carcinoma. Orphanet J Rare Dis, 2006. 1 (1): 23. 